\title{
RELAÇÃO INTENSIDADE-DURAÇÃO-FREQUÊNCIA DE CHUVAS EXTREMAS NA REGIÃO NORDESTE DO BRASIL
}

\author{
SILVA, Camila Bittencourt - camibittencourt@yahoo.com.br \\ Universidade Federal de Lavras/ UFLA \\ OLIVEIRA,Luiz Fernando Coutinho - coutinho@deg.ufla.br \\ Universidade Federal de Lavras/ UFLA
}

\begin{abstract}
RESUMO: As relações intensidade-duração-frequência (IDF) possibilitam a determinação da chuva de projeto, importante no dimensionamento de obras hidráulicas nas áreas urbanas e rurais. A escassez de estações pluviográficas levou ao desenvolvimento de metodologias que permitam a estimação da relação IDF por meio de técnicas de desagregação de chuvas utilizando dados de pluviômetros. Neste contexto, objetivaramse a geração de mapas temáticos dos parâmetros ajustados das relações IDF, para 2.042 estações pluviométricas, localizadas na região Nordeste do Brasil. Foram selecionadas as estações pluviométricas que apresentaram séries históricas com, no mínimo, 15 anos de observações diárias ininterruptas. As chuvas de 1 dia foram desagregadas, permitindo assim a geração das séries de chuvas com durações de $5,10,15,20,25,30,60,360$, 480, 600, 720 e 1440 minutos. O modelo de probabilidade de Gumbel foi empregado na análise da distribuição de frequência e na estimativa das chuvas intensas para os períodos de retorno de 5, 10, 25, 50 e 100 anos. Na verificação da aderência das frequências estimadas às observadas utilizou-se do teste do Qui-quadrado e a qualidade do ajuste dos parâmetros das relações IDF, o coeficiente de desempenho de Willmott. Para todas as estações empregadas neste estudo, os coeficientes de desempenho de Willmott apresentaram valores acima de 0,85 , conferindo um perfeito ajuste das relações IDF com coeficientes de determinação próximos de 1,0 . A constatação da dependência espacial dos parâmetros da relação IDF por meio do coeficiente de variação, permitiu a utilização da técnica de krigagem na regionalização dos valores, gerando mapas temáticos que originaram informações para locais desprovidos de monitoramento. A qualidade da superfície predita foi avaliada por meio da análise de erros gerados pela validação que seguiram uma distribuição normal, premissa básica desejável em geoestatística.
\end{abstract}

Palavras- chaves: Chuva intensa. Relação IDF. Espacialização.

INTENSITY-DURATION-FREQUENCY RELATION OF EXTREME RAINS IN THE NORTHEASTERN REGION OF BRAZIL

ABSTRACT: The intensity-duration-frequency relations (IDF) allow the determination of project rainfall, important for dimensioning hydraulic constructions in urban and rural areas. The scarcity of pluviographic stations has led to the development of methodologies that allow the estimation of the IDF relation by means of rainfall disaggregation techniques using rainfall data. In this context, the generation of thematic maps adjusted to IDF relations, for 2,042 pluviometric stations, located in the Northeastern regions of Brazil, was sought after. Pluviometric stations that presented historic series of, a minimum of, 15 years if uninterrupted daily observations were selected. The rainfall of 1 day was disaggregated, thus allowing the generation of the rainfall series with durations o $5,10,15,20,25,30,60,360,480,600,720$ and 1440 minutes. The Gumbel probability model was employed for distribution analysis of frequency and for the estimation of intense rainfall for the return periods of 5, 10, 25, 50 and 100 years. The Chi-Square test was used to verify the adherence of the estimated frequencies to those observed, and the Willmott performance coefficient was used to verify the quality of the adjustment of the IDF relation parameters. For all stations employed in this study, the Willmott performance coefficient presented values above 0.85 , conferring a perfect adjustment of the IDF relations with coefficients of determination close to 1.0. The ascertainment of special dependents of the IDF relation parameters by means of the 
coefficient of variation allowed the use of the kriging technique for regionalizing for values, generating thematic maps, which give information for locations deprived of monitoring. The quality of the predicted surface was evaluated by means of analyzing the errors generated by the validation, which followed normal distribution, basic premise desired in geostatistics.

Keywords: Intense rainfall. IDF relation. Spatialization.

\section{INTRODUÇÃO}

Dentre os processos que compõem o ciclo hidrológico, a precipitação do tipo chuva, denominada de precipitação pluvial, é fundamental para o entendimento da dinâmica do meio físico (MELLO; SILVA 2009). A precipitação pluvial apresenta irregularidade de distribuição espacial e temporal, sendo consideráveis as alterações de sua intensidade, tornando-se essencial seu conhecimento no planejamento e projeto de obras hidráulicas e agrícolas, como bueiros, barragens, irrigação, controle de inundações e erosão (Oliveira et al., 2010).

De acordo com Mello et al. (2001) a caracterização de precipitações para estudos hidrológicos é realizada conhecendo-se a intensidade, o tempo de duração e a frequência de ocorrência, permitindo, assim, definir a relação intensidade-duração-frequência (IDF) que é expressa por modelos matemáticos.

Segundo Villela e Mattos (1975), o modelo matemático clássico mais utilizado é expresso pela Equação 1.

$$
i_{m}=\frac{k T R^{a}}{(t+b)^{c}}
$$

em que:

$\mathrm{i}_{\mathrm{m}}=$ intensidade de precipitação média máxima $\left(\mathrm{mm} \mathrm{h}^{-1}\right)$;

$\mathrm{k}$, a, b e c= parâmetros de ajuste locais;

$\mathrm{TR}=$ período de retorno (ano);

$\mathrm{t}=$ tempo de duração da chuva (min)

Os trabalhos de definição das relações IDF encontram limitações decorrentes da escassez de dados pluviográficos, além de demandar um exaustivo trabalho de tabulação, análise e interpretação de grande quantidade de dados (OLIVEIRA et al., 2005). Como alternativa à baixa densidade de estações pluviográficas e em razão da ampla rede pluviométrica, foram desenvolvidas algumas técnicas de obtenção de chuva de menor duração, a partir da chuva de um dia obtida de pluviômetro. Entre essas metodologias estão a técnica das isozonas desenvolvida por Torrico (1975) e da desagregação de chuvas de 24 horas proposta pelo DAEE-CETESB (1980).

De acordo com Back (2008), o método da desagregação é de fácil utilização e fornece resultados satisfatórios, apresentando assim vantagem sobre o método das isozonas. Entretanto, Oliveira et al. (2008) avaliando o desempenho de diferentes metodologias de estimativa de chuvas intensas, compararam com as relações IDF ajustadas a partir de pluviogramas, obtendo menores desvios para o método das isozonas. Por outro lado, em estudo 
realizado por Robaina (1996) o método de desagregação apresentou desvios máximos em torno de $15 \%$, considerando a metodologia eficaz e recomendando o uso em localidades que não dispõem de registros pluviográficos.

Devido à ampla possibilidade de diferentes técnicas de estimação de chuvas intensas utilizando dados de pluviômetro, os trabalhos com a finalidade de determinar relações IDF vêm aumentando nos últimos anos. O trabalho precursor de chuvas intensas no Brasil foi o de Pfafstetter (1957); após isso, houve uma grande lacuna de trabalhos com esse enfoque, constando novas publicações somente a partir da década de 1980, com o trabalho de Denardin et al. (1980) para o Estado do Rio Grande do Sul. Na década de 1990 foram realizados os trabalhos de Fendrich (1998) para o Estado do Paraná, Martinez Junior e Magni (1999) para o Estado de São Paulo e Pinto et al. (1996) para o estado de Minas Gerais. A partir dos anos 2000 esses trabalhos começaram a se intensificar com os estudos de Davis e Naghettini (2000) para o Estado do Rio de Janeiro, Oliveira et al. $(2000,2005)$ para o Estado de Goiás e Distrito Federal; Nerilo et al. (2002) para Santa Catarina; Silva et al. (2003) para Tocantins; Santos et al. (2009) para Mato Grosso do Sul; Oliveira et al. (2011) para Mato Grosso; Fernandes et al. (2015) para o Rio de Janeiro, além do trabalho de Freitas et al. (2001) para os estados de Minas Gerais e estações limítrofes dos Estados da Bahia e Espírito Santo.

Para a região Nordeste destacam-se os trabalhos realizados por Silva et al. (2002) para a Bahia; Coutinho et al. (2010) e Silva et al. (2012) para Pernambuco; Aragão et al. (2013) para Sergipe; Fechine Sobrinho et al. (2014) para as estações de Juazeiro do Norte, Crato e Barbalha no Sul do CE e Campos et al. (2014) para o Estado do Piauí.

Dentro desse contexto, esse trabalho tem como objetivo gerar mapas temáticos dos parâmetros ajustados das relações IDF para estações pluviométricas localizadas na região Nordeste do Brasil, gerando informações para locais desprovidos de monitoramento.

\section{MATERIAL E MÉTODOS}

$\mathrm{Na}$ realização deste trabalho foram obtidas as séries históricas de precipitação de 2.042 estações pluviométricas, a partir do banco de dados disponíveis, no site da ANA (2015), por meio do sistema de informação hidrológica Hidroweb. Foram escolhidas estações que apresentaram séries históricas com, no mínimo, 15 anos de observações diárias ininterruptas. As séries que apresentaram falhas não foram utilizadas no estudo. Em seguida, para cada estação pluviométrica, foram selecionadas as precipitações máximas, para cada ano, que compõem a série histórica, obtendo assim, a série anual das chuvas máximas de 1 dia de duração. Os valores de precipitação das séries anuais foram desagregados em chuvas com durações de $24,12,10,8,6$ e 1 hora e de 30, 25, 20, 15, 10 e 5 minutos, empregando o método das relações proposto pelo DAEE-CETESB (1980), em que se aplicam os coeficientes multiplicativos apresentados na Tabela 1. Esta possibilitou a obtenção da intensidade máxima média de precipitação para cada duração, que são então submetidas à análise de distribuição de frequência. 
Tabela 1 - Coeficientes de desagregação da chuva de 1 dia

\begin{tabular}{cccc}
\hline Duração & Coeficiente & Duração & Coeficiente \\
\hline $24 \mathrm{~h} / 1 \mathrm{dia}$ & 1,14 & $30 \mathrm{~min} / 1 \mathrm{~h}$ & 0,74 \\
$12 \mathrm{~h} / 24 \mathrm{~h}$ & 0,85 & $25 \mathrm{~min} / 30 \mathrm{~min}$ & 0,92 \\
$10 \mathrm{~h} / 24 \mathrm{~h}$ & 0,82 & $20 \mathrm{~min} / 30 \mathrm{~min}$ & 0,81 \\
$8 \mathrm{~h} / 24 \mathrm{~h}$ & 0,78 & $15 \mathrm{~min} / 30 \mathrm{~min}$ & 0,70 \\
$6 \mathrm{~h} / 24 \mathrm{~h}$ & 0,72 & $10 \mathrm{~min} / 30 \mathrm{~min}$ & 0,54 \\
$1 \mathrm{~h} / 24 \mathrm{~h}$ & 0,42 & $5 \mathrm{~min} / 30 \mathrm{~min}$ & 0,34
\end{tabular}

Fonte: DAEE-CETESB (1980)

A probabilidade de ocorrência de um evento de chuva ou a superação do mesmo relaciona-se com a variação da intensidade, sendo obtida por meio da utilização de uma distribuição de probabilidade que permite a extrapolação para um período superior, em anos, ao da série histórica considerada. Para isso empregou-se a distribuição de probabilidade de Gumbel (Equação 2).

$$
F_{\text {teórica }}=1-\mathrm{e}^{-\mathrm{e}^{-\mathrm{Y}} \mathrm{TR}}
$$

em que:

Fteórica $=$ frequência acumulada de um valor extremo da série maior ou igual à magnitude de um determinado evento;

YTR = variável reduzida da distribuição de Gumbel.

Para obter o valor da variável reduzida da distribuição de Gumbel, aplicou-se a função de distribuição de frequência de Chow (Equações 3 e 4) (OLIVEIRA et al., 2000).

$$
\begin{gathered}
\mathrm{Y}_{\mathrm{TR}}=\frac{\mathrm{K}_{\mathrm{TR}}+0,45}{0,78} \\
\mathrm{~K}_{\mathrm{TR}}=\frac{\mathrm{X}-\overline{\mathrm{X}}}{\mathrm{S}}
\end{gathered}
$$

em que:

$\mathrm{KTR}$ = fator de frequência;

$\mathrm{X}=$ evento extremo no decorrer da série histórica ( $\mathrm{mm} \mathrm{h}-1)$;

$\overline{\mathrm{X}}=$ média dos valores extremos da série histórica $(\mathrm{mm} \mathrm{h}-1)$;

$\mathrm{S}=$ desvio padrão dos valores extremos da série histórica $(\mathrm{mm} \mathrm{h}-1)$.

Para cada série de valores extremos, verificou-se a aderência da distribuição de Gumbel pelo teste do Qui-quadrado ao nível de $5 \%$ de significância, e para avaliar o desempenho desse ajuste, utilizou-se o coeficiente de desempenho (C), proposto por Camargo e Sentelhas (1997), obtido pelo produto do índice de Willmott (d) com o coeficiente de correlação de Pearson ( $r$ ) (Equações 5, 6 e 7, respectivamente).

$$
C=r \times d
$$




$$
\begin{gathered}
\mathrm{d}=1-\left[\frac{\sum\left(\mathrm{F}_{\text {obs }}-\mathrm{F}_{\text {teórica }}\right)^{2}}{\sum\left(\left|\mathrm{F}_{\text {teórica }}-\overline{\mathrm{F}}_{\text {obs }}\right|+\left|\mathrm{F}_{\mathrm{obs}}-\overline{\mathrm{F}}_{\mathrm{obs}}\right|\right)^{2}}\right] \\
r=\sqrt{\frac{\sum\left(\mathrm{F}_{\text {teórica }}-\overline{\mathrm{F}}_{\text {obs }}\right)^{2}}{\sum\left(\mathrm{F}_{\mathrm{obs}}-\overline{\mathrm{F}}_{\mathrm{obs}}\right)^{2}}}
\end{gathered}
$$

em que:

$\overline{\mathrm{F}}_{\mathrm{obs}}=$ média das frequências observadas

O critério adotado, para interpretar o desempenho do ajuste da distribuição de Gumbel, empregando o índice C, está apresentado na Tabela 2.

Tabela 2 - Critérios de interpretação do índice de desempenho C.

\begin{tabular}{cc}
\hline Valor de $C$ & Desempenho \\
\hline$>0,85$ & Ótimo \\
0,76 a 0,85 & Muito bom \\
0,66 a 0,75 & Bom \\
0,61 a 0,65 & Mediano \\
0,51 a 0,60 & Sofrível \\
0,41 a 0,50 & Mau \\
$\leq 0,40$ & Péssimo \\
\hline
\end{tabular}

Fonte: Camargo e Sentelhas (1997)

Após a verificação da aderência das frequências teóricas obtidas pela distribuição de Gumbel as frequências observadas, foram estimadas as intensidades de precipitação médias máximas, para os períodos de retorno de5, $10,25,50$ e 100 anos. Para tal, a distribuição de Gumbel foi explicitada para a obtenção da intensidade de precipitação, em função do período de retorno (Equação 8).

$$
\mathrm{i}_{\mathrm{m}}=\mathrm{a}-\beta \operatorname{Ln}\left[\operatorname{Ln}\left(\frac{\mathrm{TR}}{\mathrm{TR}-1}\right)\right]
$$

em que: momentos;

"a " e $\beta=$ estimadores da distribuição de Gumbel obtidos pelo método dos

$\mathrm{TR}=$ período de retorno.

A quantidade de estações pluviométricas utilizadas nesse estudo é bastante elevada, impossibilitando a inserção, em um artigo, de todos os ajustes dos valores das relações IDF obtidas. Sendo assim, optou-se por apresentar os resultados na forma de mapas temáticos dos parâmetros 
ajustados das relações IDF, por meio da interpolação, que gera valores para locais desprovidos de monitoramento.

Portanto, de posse das relações IDF para as 2.042 estações, foi realizada a avaliação de dependência espacial dos parâmetros da equação de chuvas intensas (Equação 1), por meio do coeficiente de variação (CV), que mostra o quanto os valores da amostra variam em relação à média. Na literatura, há várias maneiras de classificar o $\mathrm{CV}$, e a utilizada neste trabalho segue o método definido por Ramos et al. (2009) que classificam como baixa variabilidade o CV abaixo de $12 \%$, média de variabilidade entre 12 e $60 \%$ e alta variabilidade acima de $60 \%$.

A conferência de dependência espacial aos dados em análise possibilita a utilização da krigagem como interpolador, devido a sua estimativa apresentar variância mínima e não tendenciosa (CARVALHO; VIEIRA 2001). Visando avaliar interpoladores, Silva et al. (2007) concluíram que a espacialização de variáveis climáticas, apresenta melhor resultado da superfície interpolada, utilizando o método da krigagem.

O manuseio da base de dados e a construção dos mapas temáticos dos parâmetros das relações IDF foram realizados pelo programa Arcgis $®$ versão 10.2 utilizando a ferramenta Geoestatistical Analyst. Esta permite a escolha do método de interpolação, no caso, pela Krigagem Ordinária, que fornece opções para a escolha do modelo teórico que se deseja empregar para o ajuste do semivariograma. Foram empregados os modelos teóricos Esférico, Exponencial e Gaussiano no ajuste do semivariograma.

Para a escolha do melhor modelo teórico a ser utilizado na espacialização dos parâmetros das relações IDF, e consequente conferência da qualidade da superfície predita, utilizou-se a técnica de validação cruzada. A qual consiste na retirada de uma estação da base de dados e seu valor é então estimado pela realização da interpolação com base no restante da amostra. Sendo essa operação repetida para todas as estações que tem os valores dos parâmetros da relação IDF conhecido. Desta maneira foi possível identificar os erros que deverão seguir uma distribuição normal, entre os valores observados e os estimados.

De acordo com Jakob e Young (2006), na análise de erros, gerados pela validação cruzada, deve-se encontrar o menor valor do erro médio quadrático, o erro médio padronizado deve ter seu valor próximo de zero, o erro quadrático médio padronizado próximo de 1 e o valor do erro padrão médio próximo do valor do erro quadrático médio.

\section{RESULTADOS E DISCUSSÕES}

Para as 2.042 estações estudadas neste trabalho, verificou-se pelo teste do Qui-quadrado que as frequências teóricas determinadas pela distribuição de Gumbel aderiram-se às frequências observadas. A Tabela 3 apresenta as estatísticas empregadas na verificação e classificação da aderência das frequências teóricas, obtidas pela distribuição de Gumbel às observadas, ou seja, teste de Qui-quadrado ( $\left.\chi^{2}\right)$, índice de Willmott, coeficientes de correlação (r) e de desempenho (d) para as capitais dos estados da região Nordeste, comportamento verificado para as 2.042 estações empregadas neste trabalho 
Tabela 3 - Estatística empregada na verificação e classificação das frequências teóricas às observadas para as capitais da Região Nordeste.

\begin{tabular}{|c|c|c|c|c|c|c|}
\hline \multirow{2}{*}{ Estação } & \multicolumn{2}{|c|}{$\chi^{2}$} & \multirow{2}{*}{$d$} & \multirow{2}{*}{$r$} & \multirow{2}{*}{ C } & \multirow{2}{*}{ Classificação } \\
\hline & Calculado & Teórico & & & & \\
\hline Maceió & 0,22 & 80,23 & 1,00 & 0,99 & 0,99 & Ótimo \\
\hline Salvador & 0,62 & 48,60 & 0,99 & 0,97 & 0,95 & Ótimo \\
\hline Fortaleza & 0,52 & 95,08 & 0,99 & 0,99 & 0,98 & Ótimo \\
\hline São Luís & 0,85 & 73,31 & 0,99 & 0,97 & 0,96 & Ótimo \\
\hline João Pessoa & 0,32 & 66,34 & 0,99 & 0,98 & 0,97 & Ótimo \\
\hline Recife & 0,22 & 44,99 & 1,00 & 0,99 & 0,99 & Ótimo \\
\hline Teresina & 0,23 & 100,75 & 1,00 & 1,00 & 0,99 & Ótimo \\
\hline Natal & 0,19 & 64,00 & 1,00 & 1,00 & 0,99 & Ótimo \\
\hline Aracaju & 0,32 & 28,87 & 0,99 & 0,99 & 0,98 & Ótimo \\
\hline
\end{tabular}

Analisando-se os valores dos coeficientes de desempenho de Camargo e Sentelhas (1997), verificam-se que a frequências teóricas determinadas pela distribuição de Gumbel se aderem as frequências observadas, com valores de c acima de 0,85. Portanto os ajustes das relações IDF podem ser classificados como ótimo para as 2.042 estações empregadas neste trabalho, permitindo, assim, as estimativas das intensidades de precipitações extremas para diferentes períodos de retorno.

A Tabela 4 apresenta os parâmetros ajustados das relações IDF para as estações localizadas nas capitais dos estados da região Nordeste com os respectivos coeficientes de determinação $\left(r^{2}\right)$. Observa-se que em todas as estações os valores dos coeficientes de determinação $(r 2)$ foram próximos de 1,0, evidenciando um perfeito ajuste das relações IDF ajustadas pela metodologia empregada, neste trabalho. Santos et al. (2009) e Silva et al. (2003) realizaram trabalhos semelhantes para os Estados de Mato Grosso e Tocantins respectivamente, obtendo coeficientes de determinação $r 2$ médios de 0,98 e 0,99, respectivamente. 
Tabela 4 - Ajuste dos parâmetros das relações IDF para as capitais da região Nordeste

\begin{tabular}{lccccccc}
\hline \multicolumn{1}{r}{ Estação } & Latitude & Longitude & $\mathrm{K}$ & $\mathrm{a}$ & $\mathrm{b}$ & $\mathrm{c}$ & $\mathrm{r}^{2}$ \\
\hline Maceió & $9^{\circ} 34^{\prime} 0^{\prime \prime}$ & $35^{\circ} 46^{\prime} 60^{\prime \prime}$ & 886,0284 & 0,1954 & 9,7839 & 0,7240 & 0,99 \\
Salvador & $13^{\circ} 1^{\prime} 0^{\prime \prime}$ & $38^{\circ} 30^{\prime} 60^{\prime \prime}$ & 922,2271 & 0,1551 & 9,7861 & 0,7242 & 0,99 \\
Fortaleza & $3^{\circ} 44^{\prime} 0^{\prime \prime}$ & $38^{\circ} 32^{\prime} 60^{\prime \prime}$ & 861,4825 & 0,1473 & 9,7823 & 0,7241 & 0,99 \\
São Luís & $2^{\circ} 53^{\prime} 0^{\prime \prime}$ & $4^{\circ} 21^{\prime} 0^{\prime \prime}$ & 1020,2781 & 0,1249 & 9,7839 & 0,7242 & 0,99 \\
João Pessoa & $7^{\circ} 6^{\prime} 60^{\prime \prime}$ & $3^{\circ} 53^{\prime} 0^{\prime \prime}$ & 1011,8743 & 0,1846 & 9,7855 & 0,7242 & 0,99 \\
Recife & $8^{\circ} 1^{\prime} 60^{\prime \prime}$ & $3^{\circ} 53^{\prime} 60^{\prime \prime}$ & 1082,0212 & 0,1620 & 9,7820 & 0,7240 & 0,99 \\
Teresina & $5^{\circ} 5^{\prime} 16^{\prime \prime}$ & $42^{\circ} 47^{\prime} 57^{\prime \prime}$ & 826,1809 & 0,1552 & 9,7847 & 0,7242 & 0,99 \\
Natal & $5^{\circ} 48^{\prime} 0^{\prime \prime}$ & $35^{\circ} 13^{\prime} 0^{\prime \prime}$ & 944,4026 & 0,1595 & 9,7825 & 0,7241 & 0,99 \\
Aracaju & $1^{\circ} 54^{\prime} 0^{\prime \prime}$ & $3^{\circ} 2^{\prime} 60^{\prime \prime}$ & 908,3115 & 0,1997 & 9,7844 & 0,7242 & 0,99 \\
\hline
\end{tabular}

A inclusão de variáveis físicas ambientais como a chuva intensa, em um modelo estatístico, torna a sua aplicação mais complexa em razão da alta variabilidade apresentada pelo fenômeno diante do grande número de atributos envolvidos em sua dinâmica (MELLO; SILVA, 2009). Entretanto os dados obtidos nessa etapa estatística podem ser considerados excelentes, pois as verificações de desempenho de todos os testes realizados retornaram bons resultados.

Com as relações IDF ajustadas para as estações pluviométricas empregadas neste estudo, foi realizada a avaliação de dependência espacial dos parâmetros da equação de chuvas intensas por meio do coeficiente de variação (CV). A Tabela 5 apresenta a estatística descritiva dos parâmetros das relações IDF para toda a região Nordeste.

Tabela 5 - Estatística descritiva dos parâmetros das relações IDF para toda a região Nordeste.

\begin{tabular}{cccccc}
\hline Parâmetro & Mínimo & Máximo & Média & Desvio Padrão & CV (\%) \\
\hline k & 280,6237 & 1669,1108 & 764,7916 & 142,7882 & 18,67 \\
a & 0,0348 & 0,2979 & 0,1606 & 0,0271 & 16,89 \\
b & 8,9300 & 13,1124 & 9,7795 & 0,0949 & 0,97 \\
c & 0,7029 & 0,7928 & 0,7241 & 0,0021 & 0,29 \\
\hline
\end{tabular}

Os parâmetros "k" e "a" obtiveram valores que os classificam com média variação, indicando a existência de uma dependência espacial, mesmo que baixa, tal como obtido por Mello et al. (2003). Vieira, Lombardi Neto e Burrows (1991) encontraram CV, para o parâmetro "a", no Estado de São Paulo, com valores semelhantes ao encontrado neste trabalho. Esses resultados indicam, também, a relação entre os parâmetros " $k$ " e "a" com a variação das intensidades de precipitação para locais diferentes da região, pois, para ambos os parâmetros, as estações que apresentaram maiores valores apresentaram, também, maiores intensidades de chuva.

Com relação aos parâmetros "b" e "c", a variação apresentada ficou abaixo de $1 \%$, com valores do CV que os classificam com baixa variabilidade, indicando uma homogeneidade nos dados sendo possível admitir os valores médios desses parâmetros como sendo um valor regionalizado. Resultados 
semelhantes foram obtidos por Mello et al. (2003) para o Estado de São Paulo, Santos et al. (2009) para o Estado do Mato Grosso do Sul, Oliveira et al. (2011) para o Estado do Mato Grosso, Souza et al. (2012) para o Estado do Pará e Campos et al. (2014) para o Estado do Piauí.

Ainda que baixa, a dependência espacial foi detectada em todos os parâmetros, possibilitando a utilização da krigagem, contudo, a interpolação dos parâmetros das relações IDF ajustados pode apresentar resultados mais precisos conforme o método utilizado e o parâmetro interpolado. A Tabela 6 apresenta a análise dos erros gerados pela validação cruzada para a escolha do modelo teórico do semivariograma.

Tabela 6 - Análise dos erros gerados pela validação cruzada dos modelos dos semivariogramas Esférico, Exponencial e Gaussiano.

\begin{tabular}{|c|c|c|c|c|c|}
\hline 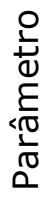 & Modelo & $\begin{array}{c}\text { Erro } \\
\text { quadrático } \\
\text { médio }^{* 1}\end{array}$ & $\begin{array}{c}\text { Erro médio } \\
\text { padronizado } 22\end{array}$ & $\begin{array}{c}\text { Erro } \\
\text { quadrático } \\
\text { médio } \\
\text { padronizado*3 }^{*}\end{array}$ & $\begin{array}{c}\text { Erro } \\
\text { padrão } \\
\text { médio*4 }\end{array}$ \\
\hline \multirow{3}{*}{$\mathrm{k}$} & Esférico & 114,5007 & $-0,0067$ & 0,8925 & 129,2083 \\
\hline & Exponencial & 114,7569 & $-0,0058$ & 0,8769 & 131,6944 \\
\hline & Gaussiano & 114,6251 & $-0,0061$ & 0,8980 & 128,6153 \\
\hline \multirow{3}{*}{$a$} & Esférico & 0,0245 & $-0,0033$ & 0,9458 & 0,025982 \\
\hline & Exponencial & 0,0244 & $-0,0025$ & 0,9452 & 0,025988 \\
\hline & Gaussiano & 0,0245 & $-0,0030$ & 0,9455 & 0,025990 \\
\hline \multirow{3}{*}{ b } & Esférico & 0,0953 & 0,00026 & 0,9130 & 0,1048 \\
\hline & Exponencial & 0,09481 & 0,00033 & 0,8993 & 0,1055 \\
\hline & Gaussiano & 0,0956 & 0,00024 & 0,9163 & 0,1049 \\
\hline \multirow{3}{*}{ C } & Esférico & 0,00209 & 0,0002 & 0,8830 & 0,002377 \\
\hline & Exponencial & 0,00208 & 0,0002 & 0,8766 & 0,002383 \\
\hline & Gaussiano & 0,00210 & 0,0001 & 0,8910 & 0,002371 \\
\hline
\end{tabular}

Pelos resultados apresentados na Tabele 6, verifica-se que para os parâmetros "k", "b" e "c", o modelo Gaussiano foi mais adequado no ajuste dos semivariogramas teóricos. Porém, para o parâmetro "a", os semivariogramas teóricos foram melhores ajustados quando se empregaram os modelos Esférico e Exponencial. Pela tendência dos menores desvios obtidos pelo modelo Gaussiano, optou- se pela sua utilização na espacialização de todos os parâmetros das relações IDF, além disso, os erros seguiram a distribuição normal, premissa básica desejável em geoestatística (MELLO et al., 2008).

A análise isolada dos parâmetros das relações IDF, não apresenta nenhum significado físico além da possibilidade de decisão de uso de valores regionais ou locais na estimativa de intensidade de chuvas (MELLO et al., 2003). Contudo a análise isolada possibilita algumas observações, sendo assim, a seguir, nas Figuras 1 a 4, são apresentados os mapas temáticos dos parâmetros 
ajustados das relações IDF, podendo observar a distribuição espacial dos valores dos parâmetros.

As regiões onde foram encontrados os maiores valores do parâmetro " $k$ " coincidem com as regiões que apresentam os maiores valores de precipitação, entre 1050 a 1800 mm, de acordo com Silva, Pereira e Almeida (2012). Podese, também, estabelecer uma relação entre os parâmetros "k" e "a" (Figuras 1 e 2) com o período de retorno, pois seus valores permitem estabelecer um vínculo com as intensidades de precipitação, ou seja, fenômenos climáticos característicos de chuvas intensas e escassez ocorrem em regiões de maiores e menores valores de ambos os parâmetros. Notam-se, ainda, coincidências com as características da classificação climática de Köppen.

Na Figura 1 pode-se observar que para o estado do Ceará, Oeste do Rio Grande do Norte e interior da Paraíba e Pernambuco, uma região central de cor azul mais escura indicando maiores valores de precipitações que podem estar relacionados com a atuação da Zona de Convergência Inter Tropical (ZCIT), bem como no Estado do Maranhão. Quando ocorre o bloqueio da atividade ciclônica no hemisfério Norte, o anticiclone dos Açores se intensifica e movimenta a ZCIT para o Sul, provocando chuvas na região (NAMIAS, 1972). Contudo valores mais altos de precipitação, no Ceará, podem estar associados, também, à atuação de sistemas frontais (KOUSKY, 1979).

A influência da ZCIT ocorre de fevereiro a maio podendo ser notada, principalmente, no Ceará, Oeste do Rio Grande do Norte e interior da Paraíba e Pernambuco (ARAÚJO et al., 2008).

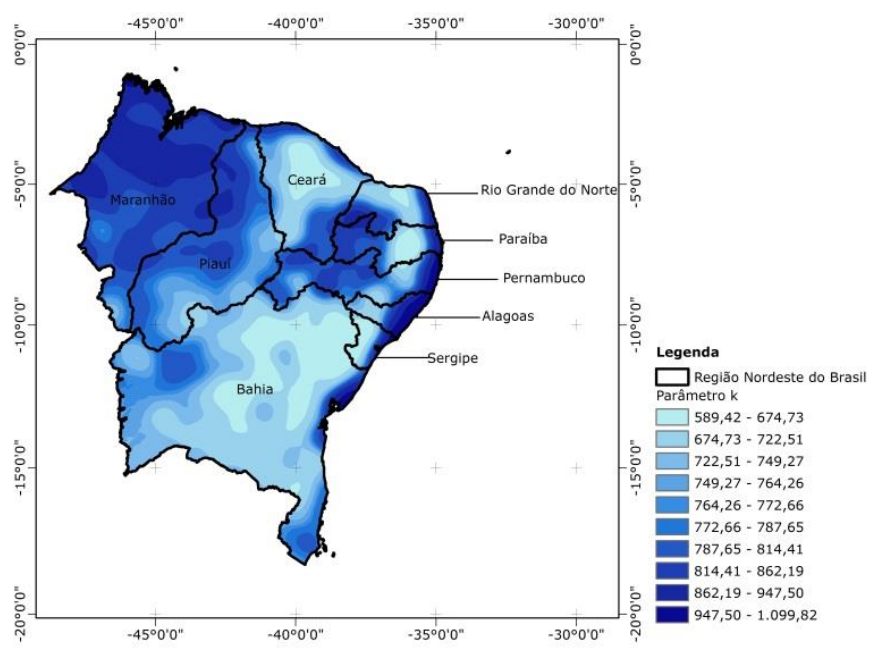

Figura 1- Superfície interpolada do parâmetro k 


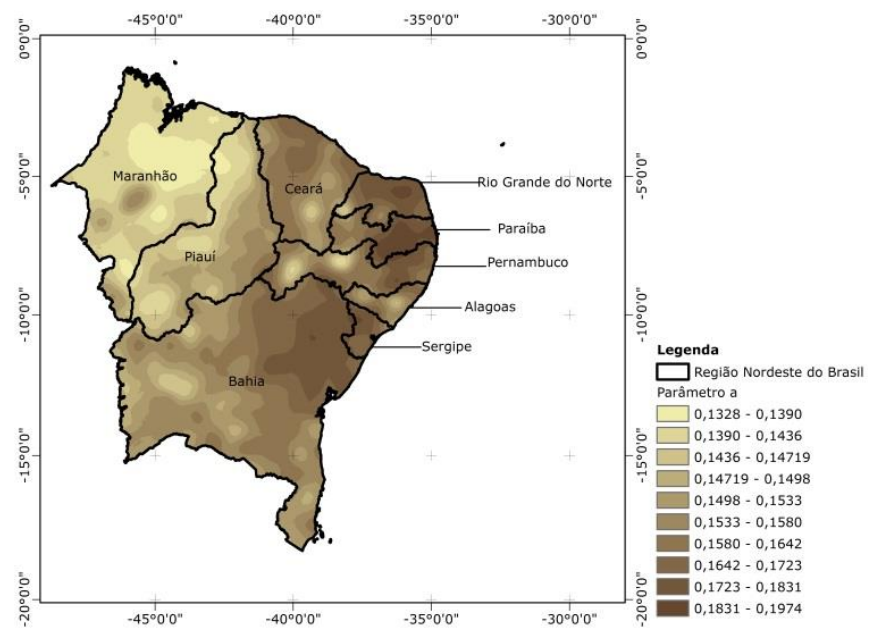

Figura 2- Superfície interpolada do parâmetro a.

Os maiores valores do parâmetro "k", observados na faixa litorânea do Piauí, Ceará e do Nordeste do Rio Grande do Norte ao Norte da Bahia, podem estar relacionados com os climas de Monção (Am) e Tropical Úmido (Af) encontrados na região, bem como à atuação dos Distúrbios de Leste. Com relação aos baixos valores do parâmetro " $k$ " observados no interior dos Estados de Alagoas, Ceará, Bahia, Pernambuco e Sergipe, estes se devem à atuação do clima Desértico (BWh) na região.

Os maiores valores do parâmetro "a" (Figura 2), no Estado da Bahia, podem ser explicados pela atuação dos sistemas frontais que têm uma frequência grande no Sul e Sudeste do Brasil; entretanto, parte deles consegue penetrar na região Nordeste provocando chuvas (ARAÚJO et al., 2008).

A presença de ilhas com características secas e entorno de valores maiores, em algumas regiões dos Estados de Alagoas, Pernambuco, Paraíba, Rio Grande do Norte, Ceará e Piauí, na Figura 2, podem ser relacionadas com o clima Semiárido (BSh) atuante na região, bem como explicada pela atuação dos vórtices ciclônicos de ar superior (VCAS) que apresentam tais características (ARAÚjO et al., 2008).

Os VCAS são encontrados de forma irregular quanto à posição, mas atuam em toda a região Nordeste, produzindo chuvas intensas e, também, grandes estiagens. O aparecimento de VCAS se relaciona, também, com a penetração de frentes frias advindas do Sul e com a posição da Zona de Convergência do Atlântico Sul (ZCAS) (ARAÚJO et al., 2008).

Durante a primavera e verão do hemisfério sul, os sistemas frontais se posicionam, na porção central da América do Sul, criando a ZCAS, uma zona de convergência de umidade que causa a estação chuvosa de forma localizada na parte Sul interior do Estado da Bahia, entre os meses de novembro a março (MOLION; BERNARDO, 2002).

Com relação aos parâmetros "b" e "c" (Figuras 3 e 4, respectivamente), pode-se observar que apresentam uma homogeneidade na distribuição espacial que reflete a baixa variação verificada pelos valores do CV (Tabela 6). 


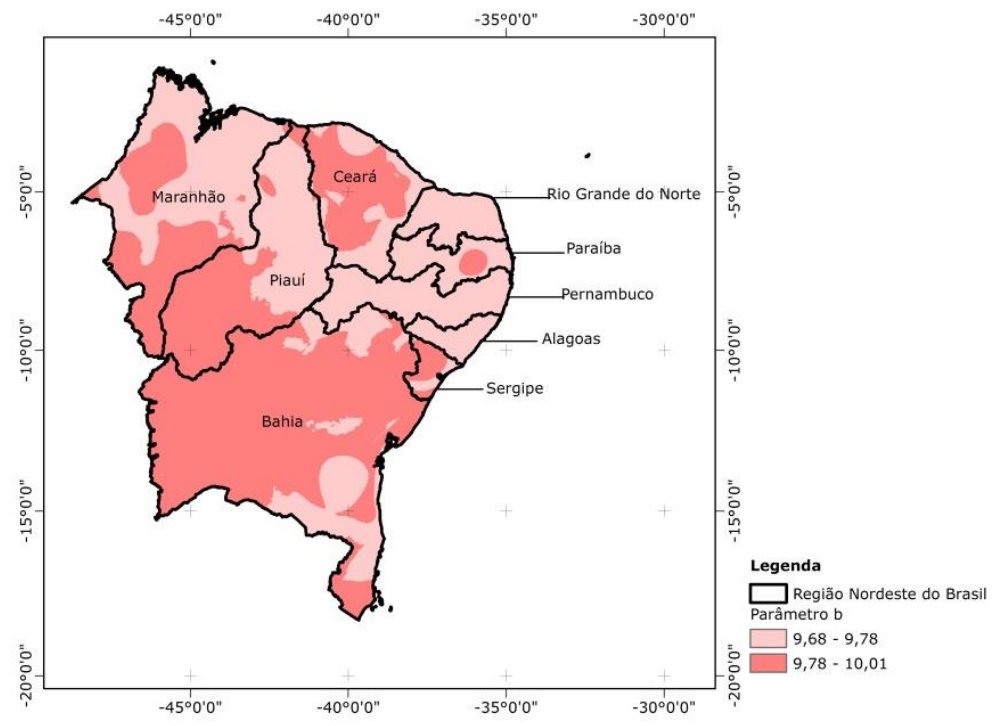

Figura 3- Superfície interpolada do parâmetro b.

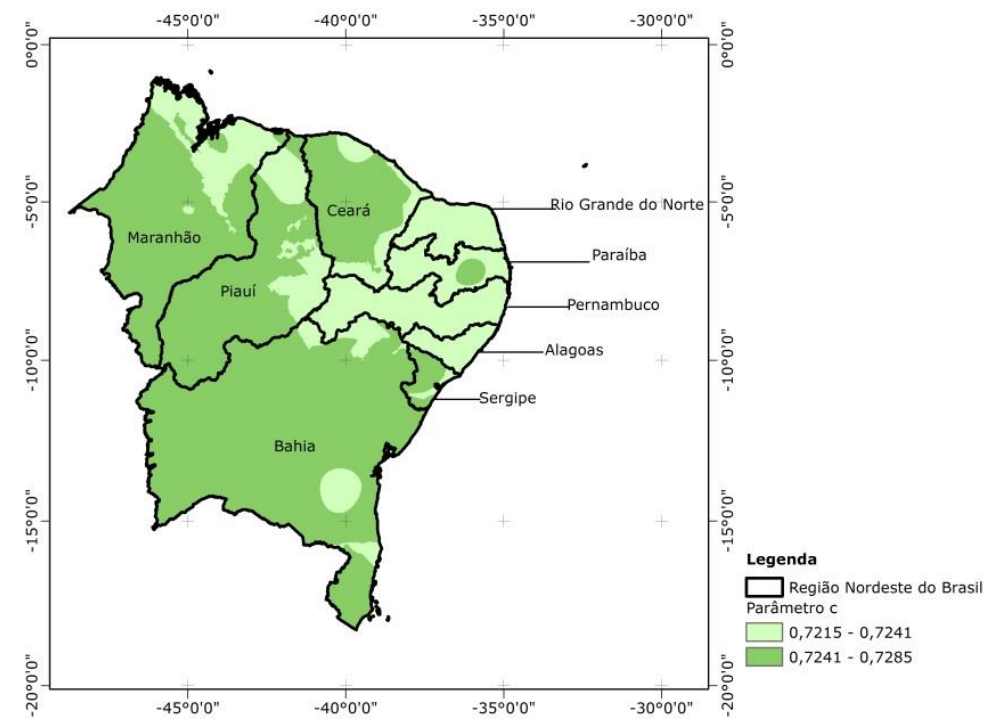

Figura 4- Superfície interpolada do parâmetro c.

Lorenzoni et al. (2014) encontraram valores constantes dos parâmetros "b" e "c" para duas estações nas cidades de Guaporema e Cidade Gaúcha no Estado do Paraná. Almeida et al. (2013) encontraram valores menos variáveis de ambos os parâmetros para os municípios de Viçosa e Palmeira dos Índios no Estado de Alagoas. Souza et al. (2012) verificaram que os parâmetros "b" e "c" apresentaram baixa variação com valores próximos da média. Aragão et al. (2013) testaram algumas metodologias para estimativas de chuvas no Estado de Sergipe, fixando os valores dos parâmetros " $b$ " e "c" e utilizando valores individuais com baixa variação, obtendo um erro de $5 \%$ entre os valores de intensidade, para as diferentes metodologias, considerando-o desprezível e 
inferindo que os parâmetros " $b$ " e " $c$ " podem ser regionalizados sem perda da qualidade dos resultados da estimação.

Apesar da pouca variação nos valores dos parâmetros " $b$ " e "c", a presença da classe mais elevada representada no Estado da Paraíba pode corresponder às altitudes mais elevadas da Serra da Borborema, podendo, dessa forma, relacionar com os valores obtidos em " $k$ ", na região litorânea, entendendo que, ao lado Leste da Serra, estão as áreas mais úmidas (Zona da Mata), já, ao Oeste, encontram-se áreas mais secas, tendo, no planalto da Borborema, um obstáculo natural para a chegada de chuvas no sertão (SILVA et al., 2011). Da mesma forma, ocorre, no Sul da Bahia, área de maior valor dos parâmetros "b" e "c" corresponde a maiores elevações da Chapada Diamantina.

Os parâmetros das relações IDF de chuvas intensas têm a sua importância pelo produto que são capazes de gerar, ou seja, a chuva intensa fornecida pela equação compostas por estes parâmetros (MELLO et al., 2003). Dessa forma, utilizaram-se os valores dos parâmetros " $k$ ", "a", "b" e "c" das estações conhecidas, para gerar a superfície interpolada com as intensidades de precipitação médias máximas para a Região Nordeste, em um período de retorno de 10 anos e intensidade de 5 minutos, valores considerados em projetos de drenagem urbana (Figura 5).

Na Figura 5 fica nítido que as maiores intensidades das precipitações ocorrem na faixa litorânea da Região Nordeste, com exceção ao estado do Maranhão que além da faixa litorânea, em $79 \%$ de seu território verificam-se intensidades entre 184,79 a $219,91 \mathrm{~mm}$. As menores intensidades foram verificadas para as regiões semi-áridas dos estados da Bahia e Ceará evidenciando as influências climáticas e orográficas descritas neste trabalho

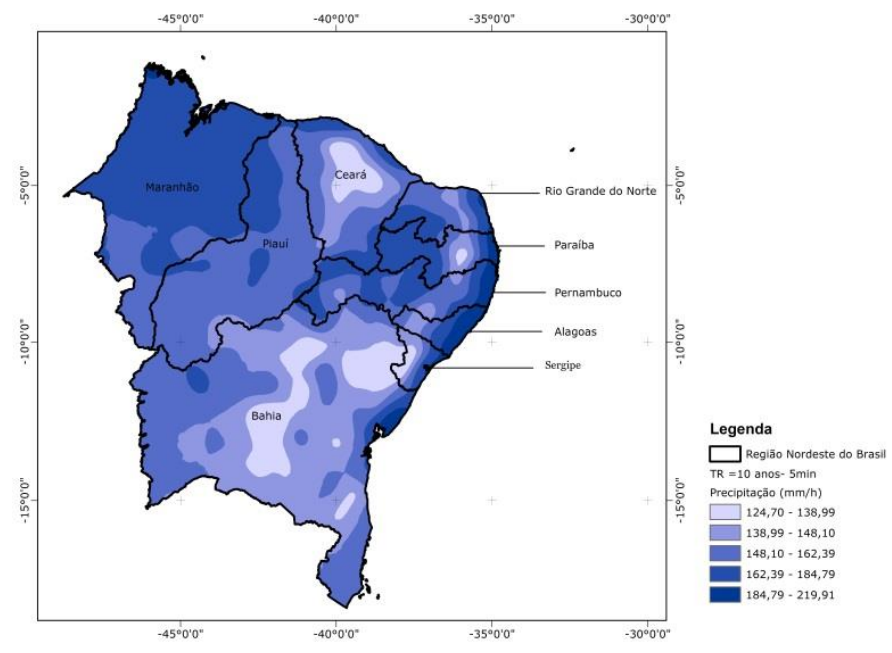

Figura 5- Precipitação média máxima $T R=10$ anos- 5 min

Com a análise dos mapas temáticos gerados, pode-se observar que há uma grande complexidade na atuação dos fatores que definem a precipitação, em cada região, podendo o mesmo sistema atmosférico provocar grandes chuvas e grandes secas, em um mesmo local, porém, em épocas diferentes. Definindo, para cada local um comportamento distinto de precipitação, ressaltando-se a importância da obtenção de relações IDF locais. 


\section{CONCLUSÃO}

A distribuição de Gumbel com parâmetros ajustados pelo método dos momentos aderiu às frequências observadas pelo teste do Qui-quadrado.

A qualidade do ajuste da distribuição de Gumbel avaliado pelo coeficiente de desempenho foi considerada como ótimo.

Foi detectada a dependência espacial dos dados, sendo possível realizar a interpolação utilizando a krigagem.

$\mathrm{Na}$ análise de erros produzidos pela validação cruzada, os valores adequados foram encontrados em todos os modelos testados, seguindo a distribuição normal, demonstrando o potencial da krigagem geoestatística para interpolação de variáveis climáticas.

Foi possível a elaboração dos mapas temáticos dos parâmetros das relações IDF para a região Nordeste do Brasil, gerando dados para áreas desprovidas de monitoramento.

Com os valores dos parâmetros das relações IDF, foi possível gerar intensidades de precipitações para o período de retorno de 10 anos com intensidade de 5 minutos, comprovando a sua utilidade na prática.

\section{REFERÊNCIAS}

ALMEIDA, K.N.S.; SOUZA, K.B.; GOMES, G.S.L.; SILVA, J.B.L.; PIRES, L.C. Parâmetros da equação de chuvas intensas nos municípios de Viçosa e Palmeira dos Índios - Al. In: CONGRESSO NORDESTINO DE ENGENHARIA FLORESTA, 4.; SEMANA DE ENGENHARIA FLORESTAL, 3., 2013, Vitória da Conquista. Anais...Vitória da Conquista, 2013. p.925-929.

ANA. AGÊNCIA NACIONAL DAS ÁGUAS. Hidro Web: sistemas de informações hidrológicas. Disponível em: http://hidroweb.ana.gov.br. Acesso em: 10 agosto 2015.

ARAGÃO, R.; SANTANA, G.R.; COSTA, C.E.F.F.; CRUZ, M.A.S.; FIGUEIREDO, E.E.; SRINIVASAN, V.S. Chuvas intensas para o Estado de Sergipe com base em dados desagregados de chuva diária. Revista Brasileira de Engenharia Agrícola e Ambiental, Campina Grande, v.17, n.3, p.243-252, 2013.

ARAÚJO, L.E.; SOUSA, F.A.S.; RIBEIRO, M.A.F.M.; SANTOS, A.S.; MEDEIROS, P.C. Análise estatística de chuvas intensas na bacia hidrográfica do Rio Paraíba. Revista Brasileira de Meteorologia, São José dos Campos, v.23, n.2, p.162-169, 2008.

BACK, A.J. Relações entre precipitações intensas de diferentes durações ocorridas no município de Urussanga, SC. Revista Brasileira de Engenharia Agrícola e Ambiental, Campina Grande, v.13, n.2, p.170-175, 2008.

BERNARDO, S. O. Clima e suas anomalias para a cidade de Maceió. 1999. 122 p. Monografia (Graduação em Física)-Universidade Federal de Alagoas, Maceió, 1999.

CAMARGO, A.P.; SENTELHAS, P.C. Avaliação do desempenho de diferentes métodos de estimativa da evapotranspiração potencial no Estado de São Paulo. Revista Brasileira de Agrometeorologia, São José dos Campos, v.5, p.89-97, 1997.

CAMPOS, A.R.; SANTOS, G.G.; SILVA, J.B.L.; IRENE FILHO, J.; LOURA, D.S. Equações de intensidade-duração-frequência de chuvas para o Estado do Piauí. 
Revista Ciência Agronômica, Fortaleza, v.45, n.3, p.488-498, 2014.

CARVALHO, J. R. P.; VIEIRA, S. R. Avaliação e comparação de estimadores de krigagem para variáveis agronômicas - uma proposta. Campinas: Embrapa Informática Agropecuária, 2001. 21 p.

COUTINHO, A.P.; SILVA, R.O.B.; SILVA, F.B.; MONTENEGRO, S.M.G.L.; ANTONINO, A.C.D. Determinação de equações de chuvas intensas para municípios das mesorregiões do Estado de Pernambuco com dados pluviométricos. In: SIMPÓSIO DE RECURSOS HÍDRICOS DO NORDESTE, 2010, Fortaleza. Anais... Fortaleza, 2010. p.1-14.

DAEE-CETESB. Departamento de Água e Energia Elétrica - Companhia de Tecnologia de Saneamento Ambiental 1980. Drenagem urbana: manual de projeto. São Paulo: DAEE-CETESB. 466 p.

DAVIS, E.G.; NAGHETTINI, M.C. Estudo de chuvas intensas no Estado do Rio de Janeiro. 2. ed. Brasília: CPRM, 2000. 50p.

DENARDIN, J.E.; FREITAS, P.L.; WÜNSCHE, W.A.; WENDT, W. Características fundamentais da chuva no Brasil: I., Rio Grande do Sul. Pesquisa Agropecuária Brasileira, v.15, n.4, p.419-421, 1980.

FECHINE SOBRINHO, V. Aplicação do método das isozonas na obtenção das Equações IDF de chuvas intensas dos municípios de Juazeiro do Norte, Barbalha e Crato, CE. 2011. 72f. Dissertação (Mestrado em Engenharia Civil)Universidade Federal do Ceará, Juazeiro do Norte, 2011.

FENDRICH, R. Chuvas intensas para obras de drenagem no Estado do Paraná. Curitiba: Champagnat, 1998. 99p.

FERNANDES, M.M.; CARVALHO, D.F.; OLIVEIRA, L.F.C.; EDUARDO, E.N. Chuvas intensas no Estado do RJ: estimativa dos parâmetros da equação intensidade duração frequência. In: CALDEIRA, M. V. W. et al. (Ed.). Tecnologia, Ciência e Extensão: como otimizar a produção florestal no Brasil?. Alegre: CAUFES, 2015. p.202-209.

FREITAS, A.J.; SILVA, D.D.; PRUSKI, F.F. Equações de chuvas intensas no Estado de Minas Gerais. Belo Horizonte: Companhia de Saneamento de Minas Gerais; Viçosa, MG: Ed. UFV, 2001. 65p.

JAKOB, A. A. E.; YOUNG, A. F. O uso de métodos de interpolação espacial de dados nas análises sócio demográficas. In: ENCONTRO NACIONAL DE ESTUDOS POPULACIONAIS, 15., 2006, Caxambu. Anais... Caxambu: ABEP, 2006. p. 2-22.

KOUSKY, V. E. Frontal influences on Northeast Brazil. Monthly Weather Review, Boston, v.107, n.103, p.1140-1153, 1979.

LORENZONI, M.Z.; PRADO, G.; SOUZA, A.H.C.; REZENDE, M.K.A.; MIOTO, L.S. Curvas intensidade-duração-frequência de chuvas intensas de Cidade Gaúcha e Guaporema, PR. In: CONGRESSO BRASILEIRO DE ENGENHARIA AGRÍCOLA, 43., 2014, Campo Grande. Anais... Campo Grande, 2014. p.1-4.

MARTINEZ JUNIOR, F.; MAGNI, N.L.G. Equações de chuvas intensas do Estado de São Paulo. São Paulo: DAEE-CTH, 1999. 124p.

MELLO, C.R.; FERREIRA, D.F.; SILVA, A.M.; LIMA, J.M. Análise de modelos matemáticos aplicados ao estudo de chuvas intensas. Revista Brasileira de 
Ciência do Solo, Viçosa, MG, v.25, n.3, p.693-698, 2001.

MELLO, C.R.; SILVA, A.M. Modelagem estatística da precipitação mensal e anual e no período seco para o Estado de Minas Gerais. Revista Brasileira de Engenharia Agrícola e Ambiental, Campina Grande, v.13, n.1, p.68-74, 2009.

MELLO, C.R.; LIMA, J.M.; SILVA, A.M.; MELLO, J.M.; OLIVEIRA, M.S. Krigagem e inverso do quadrado da distância para interpolação dos parâmetros da equação de chuvas intensas. Revista Brasileira de Ciência do Solo, Viçosa, MG, v.27, p.925-933, 2003.

MELLO, C.R.; VIOLA,M.R.; MELLO, J.M. \& SILVA, A.M. Continuidade espacial de chuvas intensas no Estado de Minas Gerais. Ciência e Agrotecnologia, 32:532539, 2008.

NAMIAS, J. Influence of northern hemisphere general circulation on drought in northeast Brazil. Tellus, Stockholm, v.24, n.4, p.336-343, 1972.

MOLION, L. C. B.; BERNARDO, S. O. Uma revisão da dinâmica das chuvas no nordeste brasileiro. Revista Brasileira de Meteorologia, São José dos Campos, v. 17, n. 1, p. 1-10, 2002.

NERILO, N.; MEDEIROS, P.A.; CORDERO, A. Chuvas intensas no Estado de Santa Catarina. Florianópolis: UFSC/EDIFURB, 2002. 156p.

OLIVEIRA, J.R.; PINTO, M.F.; SOUZA, W.J.; GUERRA, J.G.M.; CARVALHO, D.F. Erosão hídrica em um Argissolo Vermelho-Amarelo, sob diferentes padrões de chuva simulada. Revista Brasileira de Engenharia Agrícola e Ambiental, Campina Grande, v.14, n.2, p.140-147, 2010.

OLIVEIRA, L.F.C.; CORTÊS, F.C.; BARBOSA, F.O.A.; ROMÃO, P.A.; CARVALHO, D.F. Estimativa das equações de chuvas intensas para algumas localidades no Estado de Goiás pelo método da desagregação de chuvas. Pesquisa Agropecuária Tropical, Goiânia, v.30, n.1, p.23-27, 2000.

OLIVEIRA, L.F.C.; CORTÊS, F.C.; WEHR, T.R. BORGES, L.B.; SARMENTO, P.H. L.; GRIEBELER, N.P. Intensidade-duração-frequência de chuvas intensas para localidades no Estado de Goiás e Distrito Federal. Pesquisa Agropecuária Tropical, Brasília, DF, v.35, n.1, p.13-18, 2005.

OLIVEIRA, L.F.C. VIOLA, M.R.; PEREIRA, S.; MORAIS, N.R. Modelos de predição de chuvas intensas para o Estado do Mato Grosso, Brasil. Revista Ambiente e Água, Taubaté, v.6, n.3, p.274-290, 2011.

OLIVEIRA, L.F.C.; ANTONINI, J.C.A.; FIOREZE, A.P.; SILVA, M.A.S. Métodos de estimativa de precipitação máxima para o Estado de Goiás. Revista Brasileira de Engenharia Agrícola e Ambiental, Campina Grande, v.12, n.6, p.620-625, 2008.

PFAFSTETTER, O. Chuvas intensas no Brasil. Brasília, DF: Departamento Nacional de Obras e Saneamento, 1957. 246p.

PINTO, F.A.; FERREIRA, P.A.; PRUSKI, F.F.; ALVES, A.R.; CECON, P.R. Estimativa de chuvas intensas no Estado de Minas Gerais utilizando registros diários. Revista Engenharia Agrícola, Jaboticabal, v.16, n.2, p.8-21, 1996.

RAMOS, C.M.C.; SILVA, A.F.; BASSOI, L.H.; SARTORI, A.A.C.; ZIMBACK, C.R.L. Análise temporal de fatores climáticos em Petrolina, PE. In: CONGRESO ARGENTINO DE LNGENIERÍA RURAL, 10.; CONGRESO DEL MERCOSUR, 2., 
2009, Rosário. Actas...Rosário: Editorial de La Universidad Nacional de Rosario, 2009. p.1036-1041.

ROBAINA, A.D. Modelo para geração de chuvas intensas no Rio Grande do Sul. Revista Brasileira de Agrometeorologia, Santa Maria, v.4, n.2, p.95-98, 1996.

SANTOS, G.G.; FIGUEIREDO, C.C.; OLIVEIRA, L.F.C.; GRIEBELER, N.P. Intensidade-duração-frequência de chuvas para o Estado de Mato Grosso do Sul. Revista Brasileira de Engenharia Agrícola e Ambiental, Campina Grande, v.13, p.899-905, 2009.

SILVA, D.D.; GOMES FILHO, R.R.; PRUSKI, F.F.; PEREIRA, S.B.; NOVAES, L.F. Chuvas intensas no Estado da Bahia. Revista Brasileira de Engenharia Agrícola e Ambiental, Campina Grande, v.6, n.2, p.362-367, 2002.

SILVA, B.M.; MONTENEGRO, S.M.G.L.; SILVA, F.B.; ARAÚJO FILHO, P.F. Chuvas intensas em localidades do Estado de Pernambuco. Revista Brasileira de Recursos Hídricos, Porto Alegre, v.17, n.3, p.135-147, 2012.

SILVA, K. R.; PAIVA, Y. G.; CECÍLIO, R. A.; PEZZOPANE, J. E. M. Avaliação de interpoladores para a espacialização de variáveis climáticas na bacia do rio Itapemirim-ES. In: SIMPÓSIO BRASILEIRO DE SENSORIAMENTO REMOTO, 13., 2007, Florianópolis. Anais..., Florianópolis: INPE, 2007. p. 3141-3146.

SILVA, V. P. R.; PEREIRA, E. R. R.; ALMEIDA, R. S. R. Estudo da variabilidade anual e intra-anual da precipitação na região Nordeste do Brasil. Revista Brasileira de Meteorologia, São José dos Campos, v. 27, n. 2, p. 163-172, 2012.

SILVA, D.D.; PEREIRA, S.B.; PRUSKI, F.F.; GOMES FILHO, R.R.; LANA, A.M.Q.; BAENA, L.G.N. Equações de intensidade-duração-frequência da precipitação pluvial para o Estado de Tocantins. Engenharia na Agricultura, Viçosa, MG, v.11, n.4, p.1-8, 2003.

SILVA, V.P.R.; PEREIRA, E.R.R.; AZEVEDO, P.; SOUSA, F.A.S.; SOUSA, I.F. Análise da pluviometria e dias chuvosos na região Nordeste do Brasil. Revista Brasileira de Engenharia Agrícola e Ambiental, Campina Grande, v.15, n.2, p.131-138, 2011.

SOUZA, R.O.R.M.; SCARAMUSSA, P.H.M. AMARAL, M.A.C.M.; PEREIRA NETO, J.A.; PANTOJA, A.V.; SADECK, L.W.R. Equações de chuvas intensas para 0 Estado do Pará. Revista Brasileira de Engenharia Agrícola e Ambiental, Campina Grande, v.16, n.9, p.999-1005, 2012.

TORRICO, J. J. T. Práticas hidrológicas. Rio de Janeiro: Transcom, 1975. 120p.

VIEIRA, S.R.; LOMBARDI NETO, F.; BURROWS, I.T. Mapeamento da chuva diária máxima provável para o Estado de São Paulo. Revista Brasileira de Ciências do Solo, Viçosa, MG, v.15, p.93-98, 1991.

VILLELA, S.M.; MATTOS, A. Hidrologia aplicada. São Paulo: McGraw-Hill do Brasil, 1975. 245p 